\title{
Charge trapping and detrapping in polymeric materials
}

\author{
George Chen ${ }^{\mathrm{a})}$ and Zhiqiang Xu \\ School of Electronics and Computer Science, University of Southampton, Southampton, Hampshire SO16 \\ 1BJ, United Kingdom
}

(Received 21 September 2009; accepted 13 November 2009; published online 30 December 2009)

\begin{abstract}
Space charge formation in polymeric materials can cause some serious concern for design engineers as the electric field may severely be distorted, leading to part of the material being overstressed. At the worst, this may result in material degradation and possibly premature failure. It is therefore important to understand charge generation, trapping, and detrapping processes in the material. In the present paper, the characteristics of charge trapping and detrapping in low density polyethylene under dc electric field have been investigated using the pulsed electroacoustic technique. It has been found that the charge decay shows very different characteristics for the sample with different periods of electric field application. To explain the results a simple trapping and detrapping model based on two trapping levels has been proposed. Qualitative analysis revealed the similar features to those observed experimentally. (C) 2009 American Institute of Physics. [doi:10.1063/1.3273491]
\end{abstract}

\section{INTRODUCTION}

A thorough understanding the dielectric properties of polymeric insulating materials is important to efficiently and safely utilize the materials in both power and electronic industries. Charge dynamics play a crucial role in determining electrical performance of dielectrics. The development in space charge mapping techniques ${ }^{1}$ in past few decades has resulted in an abundant knowledge resource on space charge in solid dielectrics. Space charge becomes an important theme at various dielectric meetings. Recently, the phenomenon of charge trapping and detrapping has attracted significant attention. ${ }^{2,3}$ It has been demonstrated that the space charge phenomenon may be closely related to the aging taken place in the material. However, both trapping and detrapping in solid dielectrics are complex topics as they are related to microstructure of the material. Further studies are required before the relationship between morphology and traps can be established. One of the techniques to characterize traps is to investigate the time characteristics of charge trapping and detrapping.

In the present paper, a theoretical model based on shallow and deep traps has been proposed. Charge dynamics considering both trapping and detrapping have been obtained. A qualitative analysis has been carried out to study features associated with trapping and detrapping of charge in the dielectrics. To support our proposed model, we have measured charge buildup following the voltage application and charge decay after the removal of the applied voltage using the pulsed electroacoustic technique. ${ }^{4}$ The samples used were commercially available additive-free low density polyethylene (LDPE). The thickness of the sample is $\sim 180 \mu \mathrm{m}$. Space charge profiles were measured at room temperature under different applied voltages.

\section{THEORY}

Trapping and detrapping are closely related to the trap distribution in terms of spatial and energy. For a semicrys-

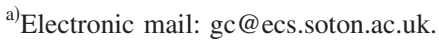

talline polymeric material, it is possible that traps may have several discrete energy depths or even continuous energy distribution. Spatially, trap distribution may vary for a sample as the material may experience different processes including physical, thermal, and chemical treatment. This is especially true for the region near to the surface. To simplify the mathematics involved here we assume that the traps are uniformly distributed across the sample and only have two trap energy levels, i.e., one representing shallow trap and another for deep trap.

\section{A. Injection process}

Charge carriers in solid dielectrics can be generated via charge injection from the electrodes and ionization within the material. From our early experimental evidence ${ }^{5}$ on the same material it is likely that the charges are injected from the electrodes. There are several processes that can lead to the current injection such as Schottky injection ${ }^{6}$ and tunneling. ${ }^{7}$ Generally speaking, the current increases exponentially with the applied field and decays exponentially with the time. The time effect can be explained in terms of the space charge field. The field near to the electrode will decrease due to the formation of trapped space charge. The reduction in local electric field results in a reduced current injection. In the present study, the injected current density is represented by the following expression:

$$
J(E, t)=J_{0} \exp \left(\frac{E}{E_{0}}\right)\left[1-\exp \left(-\frac{t}{\tau}\right)\right],
$$

where $J_{0}$ is the initial current density when the applied field is $E_{0}, E$ is the applied field, and $\tau$ is the time constant related to overall trapping characteristic of the material.

\section{B. Trapping process}

Depending on the type of defects, traps may capture electrons or holes that have been injected into (or generated within) the material under the application of electric field. It 


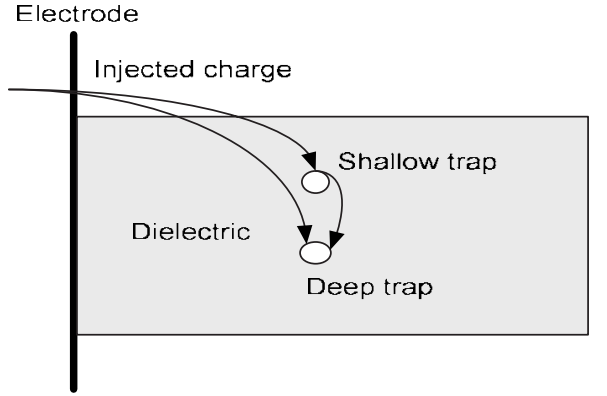

FIG. 1. The model of injected charges and their trapping processes.

has been suggested that some defects can trap both electron and hole although the trap may have different energy levels for each type of charge carrier. ${ }^{8}$

There have been a series of attempts to understand trapping behavior over the years as the formation of space charge in solid dielectrics has been considered as a key component to understand ageing phenomenon in the insulating materials. ${ }^{9-11}$ Such investigation has been intensified due to the significant development and progress in space charge measurement techniques. Several models ${ }^{12-14}$ have been proposed to simulate the formation and decay of space charge with certain degree of success. However, due to many parameters introduced in these models the exact physical processes of trapping and detrapping are not detailed. In the present study we propose a simple two levels of trap, as shown in Fig. 1.

To simplify the mathematical treatment, the trapping process from shallow to deep trap is not considered in the present study. In addition, we assume the trapped charges are close to the injecting electrode, so the recombination with the injected charge from the opposite electrode can be neglected.

Assume that $N_{1}$ and $N_{2}$ are the total number of shallow and deep traps, $\sigma_{1}$ and $\sigma_{2}$ are trapping cross section for shallow and deep trap, and the trapped charges in shallow and deep trap are represented by $n_{1}$ and $n_{2}$, respectively. The following equations may represent charge trapping dynamics

$$
\begin{aligned}
& \frac{d n_{1}}{d t}=\frac{J \sigma_{1}}{q}\left(N_{1}-n_{1}\right), \\
& \frac{d n_{2}}{d t}=\frac{J \sigma_{2}}{q}\left(N_{2}-n_{2}\right),
\end{aligned}
$$

where $q$ is the charge amount.

\section{Detrapping process}

When the applied field is removed the amount of trapped charges decreases with time, known as charge decay. Trapped charge carriers can release from the traps by several mechanisms such as photon assisted detrapping, phonon assisted (thermal) detrapping, impact ionization, and tunneling. ${ }^{15}$ Photon assisted detrapping is negligible in the present study as the sample was not exposed to any light. Impact ionization and tunneling occurs mainly at high electric fields and their influence on the detrapping is not consid-

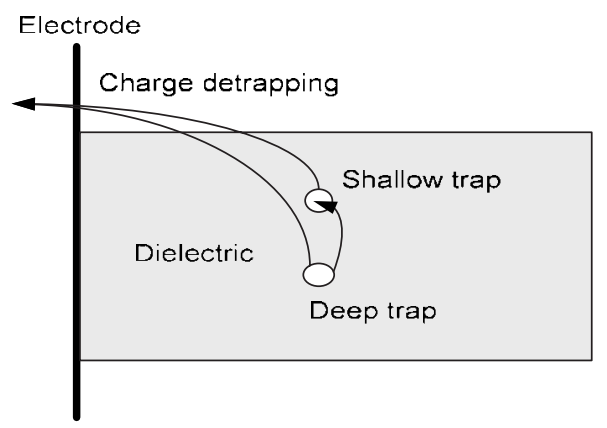

FIG. 2. Model for charge detrapping processes.

ered in the present study. Thermal detrapping occurs when the trapped charge carrier receives its energy from the thermal lattice vibrations (Fig. 2).

Again, the process of detrapping from deep to shallow trap is not considered in the first instance. The mathematical expression of the process can be described as

$$
\begin{aligned}
& \frac{d n_{1}}{d t}=-k_{1} n_{1}, \\
& \frac{d n_{2}}{d t}=-k_{2} n_{2},
\end{aligned}
$$

where $k_{1}$ and $k_{2}$ are the thermal detrapping rate constants for shallow and deep traps, respectively. The thermal detrapping rate constant can be expressed as

$$
k_{\mathrm{th}}=N_{c} \nu_{\mathrm{th}} \sigma_{c} \exp \left(-\frac{E_{t}}{k T}\right),
$$

where $N_{c}$ is the effective density of states in the conduction band, $\nu_{\text {th }}$ is the thermal velocity of the charge, $E_{t}$ is the trap depth, $k$ is the Boltzmann constant, and $T$ is the temperature.

\section{Kinetics of trapping and detrapping}

Based on the above described trapping and detrapping processes, the rate of change in the density of filled shallow trap and deep trap is thus given by

$$
\begin{aligned}
& \frac{d n_{1}}{d t}=\frac{J \sigma_{1}}{q}\left(N_{1}-n_{1}\right)-k_{1} n_{1}, \\
& \frac{d n_{2}}{d t}=\frac{J \sigma_{2}}{q}\left(N_{2}-n_{2}\right)-k_{2} n_{2} .
\end{aligned}
$$

The total rate of change in the trapped charge is the sum of the above equations, i.e.,

$$
\begin{aligned}
\frac{d n}{d t}= & \frac{d n_{1}}{d t}+\frac{d n_{2}}{d t}=\frac{J \sigma_{1}}{q}\left(N_{1}-n_{1}\right)-k_{1} n_{1}+\frac{J \sigma_{2}}{q}\left(N_{2}-n_{2}\right) \\
& -k_{2} n_{2}
\end{aligned}
$$

This equation applies when the sample is under electric field. When the electric field is removed, the thermal detrapping will be the only process governs the charge decay. The total rate of change in the trapped charge is given by 


$$
\frac{d n}{d t}=\frac{d n_{1}}{d t}+\frac{d n_{2}}{d t}=-k_{1} n_{1}-k_{2} n_{2}
$$

\section{QUALITATIVE ANALYSIS}

The above equations are standard first order deferential equations. To solve them, we need to know the initial conditions. For Eqs. (7) and (8), both $n_{1}$ and $n_{2}$ before the injection takes place should be zero, i.e.,

$$
\begin{aligned}
& n_{1}(x, 0)=0, \\
& n_{2}(x, 0)=0 .
\end{aligned}
$$

No analytical solutions can be found for Eqs. (7) and (8) if the time dependent current injection is considered. To simplify solutions we assume that the injected current depends on the applied field only, i.e., the equation becomes

$$
J(E)=J_{0} \exp \left(\frac{E}{E_{0}}\right)
$$

Now Eqs. (7) and (8) reduce to

$$
\begin{aligned}
& \frac{d n_{1}}{d t}=A_{1}\left(N_{1}-n_{1}\right)-k_{1} n_{1}, \\
& \frac{d n_{2}}{d t}=A_{2}\left(N_{2}-n_{2}\right)-k_{2} n_{2},
\end{aligned}
$$

where

$$
A_{1}=\frac{J_{0} \sigma_{1}}{q} \exp \left(\frac{E}{E_{0}}\right)
$$

and

$$
A_{2}=\frac{J_{0} \sigma_{2}}{q} \exp \left(\frac{E}{E_{0}}\right) .
$$

Considering the initial conditions for $n_{1}$ and $n_{2}$, the solutions for Eqs. (12) and (13) are

$$
\begin{aligned}
& n_{1}(t)=\frac{A_{1} N_{1}}{A_{1}+k_{1}}\left\{1-\exp \left[-\left(A_{1}+k_{1}\right) t\right]\right\}, \\
& n_{2}(t)=\frac{A_{2} N_{2}}{A_{2}+k_{2}}\left\{1-\exp \left[-\left(A_{2}+k_{2}\right) t\right]\right\} .
\end{aligned}
$$

The total trapped charge density during the application of electric field is given as

$$
\begin{aligned}
n(t)= & n_{1}(t)+n_{2}(t)=\frac{A_{1} N_{1}}{A_{1}+k_{1}}\left\{1-\exp \left[-\left(A_{1}+k_{1}\right) t\right]\right\} \\
& +\frac{A_{2} N_{2}}{A_{2}+k_{2}}\left\{1-\exp \left[-\left(A_{2}+k_{2}\right) t\right]\right\} .
\end{aligned}
$$

The detrapping processes are much simpler after the removal of the applied field. The solutions for Eqs. (4) and (5) are given below,

$$
n_{1}(t)=n_{10} \exp \left(-k_{1} t\right)
$$

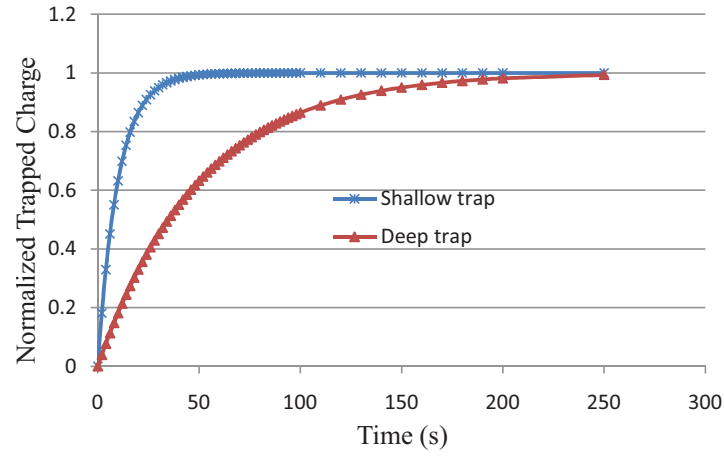

FIG. 3. (Color online) Dynamics of charge trapping for shallow and deep traps.

$$
n_{2}(t)=n_{20} \exp \left(-k_{2} t\right)
$$

where $n_{10}$ and $n_{20}$ are the initial conditions and they can be obtained from Eqs. (14) and (15) at the moment when the applied field is removed.

The total trapped charge density during detrapping process is given by

$$
n(t)=n_{1}(t)+n_{2}(t)=n_{10} \exp \left(-k_{1} t\right)+n_{20} \exp \left(-k_{2} t\right) .
$$

For trapping process, if we assume $\sigma_{1}>\sigma_{2}$, this leads $A_{1}$ $>A_{2}$. Since the deep trap depth $E_{t 2}>E_{t 1}$, using the Eq. (6) we conclude $k_{2}<k_{1}$.

Therefore, we have $\left(A_{1}+k_{1}\right)>\left(A_{2}+k_{2}\right)$, indicating that the time constant $(\tau)$ for filling the shallow traps $\left(\tau_{1}\right)$ is shorter than that of filling the deep traps $\left(\tau_{2}\right)$. To illustrate its effect, a schematic diagram where $\tau_{1}=10 \mathrm{~s}$ and $\tau_{2}=5 \tau_{1}$ is shown in Fig. 3.

The figure demonstrates that the injected charges fill shallow traps faster and deep traps slower. The exact trapped charge density in shallow traps and deep traps depends also on the total number of shallow and deep trap density. The ratio is related to the duration of the applied voltage. When the electric field is applied for a short period of time, the ratio of filling the shallow traps is greater.

For detrapping process, it is much simpler and for the same assumption we have $k_{2}<k_{1}$, indicating charge decay faster from shallow traps than that from deep traps. By examining the expression for the trapping time constant, it is reasonable to assume that the detrapping time constant from shallow traps should be larger. Figure 4 gives a similar illustration diagram of trapped charge dynamics after the removal of the applied field where $k_{1}=20 \mathrm{~s}$ and $k_{1}=5 \mathrm{k}_{2}$.

It is clear that the decay rate of charge from the shallow traps is much fast that that from deep traps.

The trapping time constant will also be affected by the applied field as one can see from expressions for $A_{1}$ and $A_{2}$. As the applied field increases the value A increases, leading to a decrease in trapping time constant. This means that the filling rate of both shallow and deep traps will increase with the applied field. The temperature will have a significant effect on both trapping and detrapping. As temperature in- 


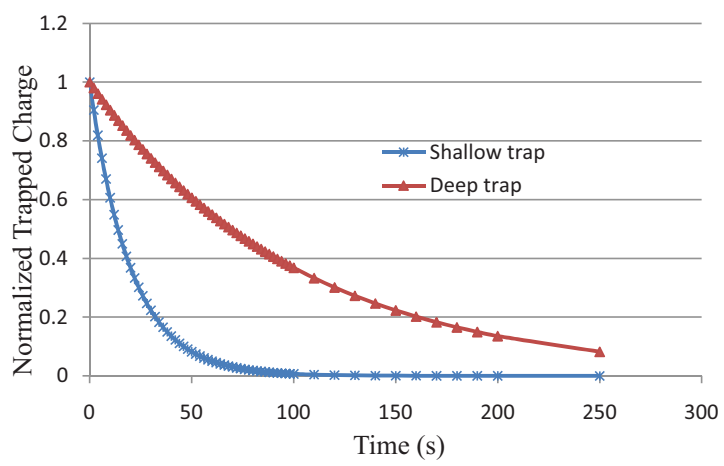

FIG. 4. (Color online) Dynamics of trapped shallow and deep charge during decay.

creases the thermal detrapping rate constant increases and this will lead to increase in both trapping rate and detrapping rate.

\section{EXPERIMENTAL RESULTS FROM LDPE}

To verify the proposed model, space charge measurements were carried out on additive-free LDPE. Considering the assumption of no recombination between the injected electrons and holes, the applied dc voltage and its duration were limited so both positive and negative charges in the sample were well separated.

Figure 5 illustrates space charge profiles and its decay for different stressing times. The sample was stressed at 4 $\mathrm{kV}$. It can be seen for 2 min stressing, the injected negative

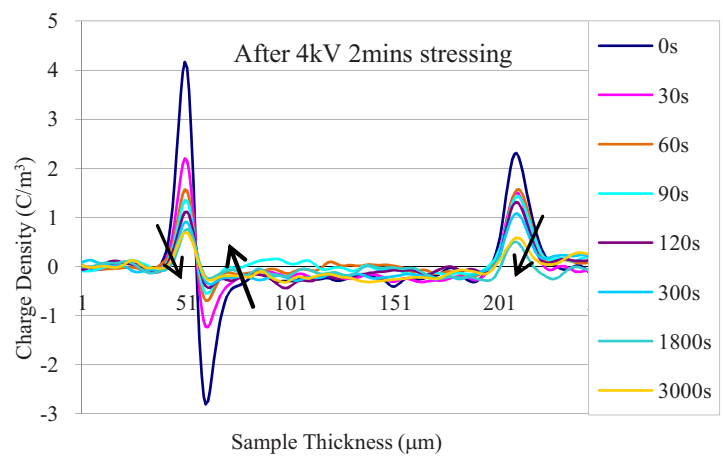

(a)

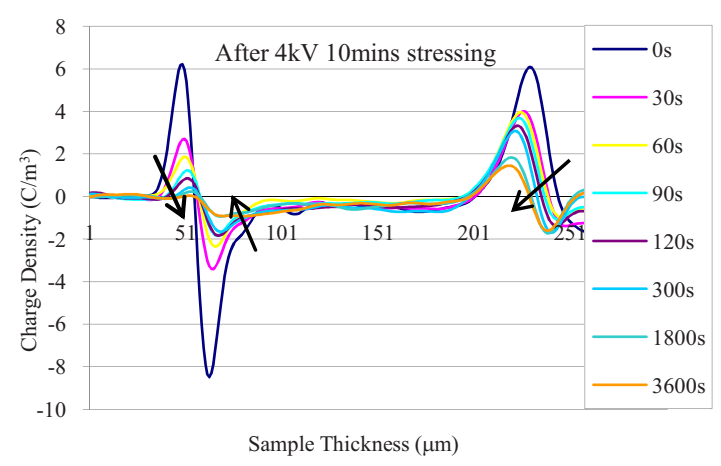

(b)

FIG. 5. (Color online) Space charge decay after the removal of the applied voltage $4 \mathrm{kV}$ at stressing duration of (a) $t=2 \mathrm{~min}$ and (b) $\mathrm{t}=10 \mathrm{~min}$.

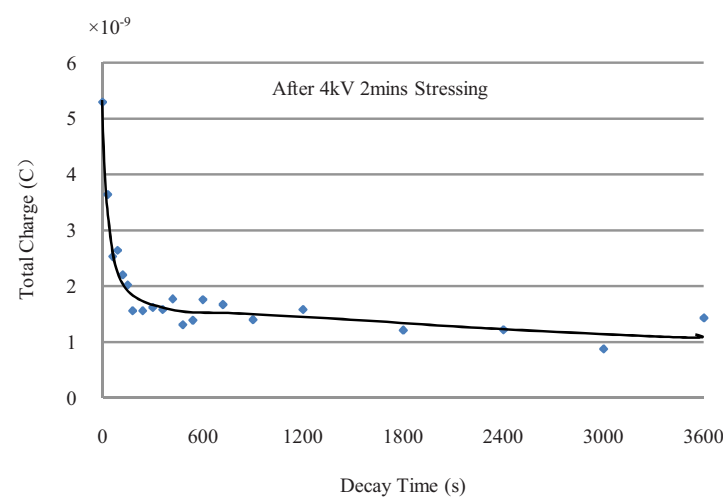

(a)

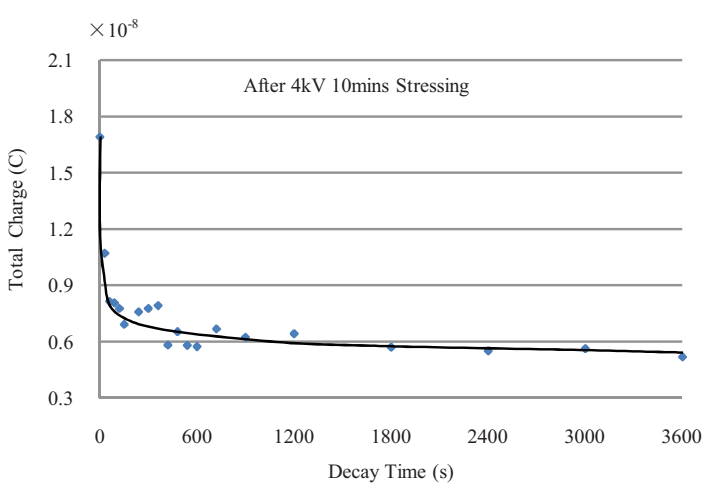

(b)

FIG. 6. (Color online) Decay of the total trapped charge after being stressed at $4 \mathrm{kV}$ for (a) $\mathrm{t}=2 \mathrm{~min}$ and (b) $\mathrm{t}=10 \mathrm{~min}$.

charge dominates bulk charge and the total charge decay is fairly fast. When the stressing time is extended to $10 \mathrm{~min}$, both positive and negative charges are present in the sample. Apart from the initial rapid decay the remaining charge decreases with a much slow rate.

The amount of charge in the bulk can be estimated using the following equation

$$
Q=\int_{0}^{d}|\rho(x)| \cdot S \cdot d x,
$$

where $\rho(x)$ is charge density, $S$ is the electrode area, and $d$ is the thickness of the sample. Since the decay of positive and negative charge shows a similar trend. In the present study the total charge is calculated.

Figure 6 shows the total charge and its relationship with time after a $4 \mathrm{kV}$ applied voltage was removed at 2 and 10 min, respectively. First of all, the total trapped charge decay in both cases shows a fast decay initially and followed a much slow change. This fact approves the two trapping levels approximation used in our analysis. The results also show that the amount of charges trapped in deep trap increases with the duration of the applied voltage. This feature is consistent with qualitative analysis in Sec. III.

Numerical simulation is required if one wants to extract parameters from the model. Trap depths and concentration of both shallow and deep traps are important as they are poten- 
tially related to microstructure of the material. Changes in parameters may reflect aging taken place in the material. It has been reported ${ }^{2,3}$ that the apparent mobility can be used as aging marker and it will certainly affected by the changes in trap depth and concentration in a material.

\section{CONCLUSIONS}

A charge trapping and detrapping model based on two trapping levels has been proposed in the present paper. By assuming the injected charges are in the vicinity of the electrodes, recombination can be neglected. Additionally, it is assumed that charge decay of the trapped charges is only influenced by the thermal process.

Qualitative analysis shows that it is easier to fill shallow traps. The trapping process is influenced by the duration and magnitude of the applied electric field. The duration of the applied field will alter the ratio of charges trapped in shallow and deep traps while the magnitude of the applied field changes trapping rate as well as amount of trapped charges. Temperature is another factor affecting the amount of trapped charge via detrapping process. Higher temperature leads to fast detrapping process.

Experimental evidence from charge decay measurements in LDPE sample revealed a rapid decay initially followed by a much slower decrease, indicating the presence of two trapping levels proposed in the model. Features from qualitative analysis of the model are largely observed in the experiment.
It is possible to extract more information from the proposed model such as trap depth and concentration of both shallow and deep traps. This can be done via a series of space charge measurements with different duration of the allied field at different field levels. By adjusting the parameters in the proposed model simulations can be carried out to match the experimental results.

${ }^{1}$ N. H. Ahmed and N. N. Srinivas, IEEE Trans. Dielectr. Electr. Insul. 4, 644 (1997).

${ }^{2}$ G. Chen and T. Van Nguyen, CMD2008, Beijing, China, 2008 (unpublished), pp. 633-637.

${ }^{3}$ G. Mazzanti, G. C. Montanari, and J. M. Alison, IEEE Trans. Dielectr. Electr. Insul. 10, 187 (2003).

${ }^{4}$ G. Chen, Y. L. Chong, and M. Fu, Meas. Sci. Technol. 17, 1974 (2006).

${ }^{5}$ G. Chen, Y. Tanaka, T. Takada, and L. Zhong, IEEE Trans. Dielectr. Electr. Insul. 11, 113 (2005).

${ }^{6}$ K. C. Kao and W. Hwang, Electrical Transport in Solids (Pergamnon, Oxford, 1981).

${ }^{7}$ S. M. Sze and K. K. Ng, Physics of Semiconductor Devices (Wiley, Hoboken, NJ, 2006).

${ }^{8}$ D. R. Wolters and J. J. Schoot, J. Appl. Phys. 58, 831 (1985).

${ }^{9}$ G. Chen, M. Fu, X. Z. Liu, and L. S. Zhong, J. Appl. Phys. 97, 083713-1, 2005.

${ }^{10}$ G. Chen, Trans. Electr. Electron. Mater. 7, 235 (2006).

${ }^{11}$ G. Mazzanti, G. C. Montanari, and L. A. Dissado, IEEE Trans. Dielectr. Electr. Insul. 6, 864 (1999).

${ }^{12}$ J. M. Alison and R. M. Hill, J. Phys. D: Appl. Phys. 27, 1291 (1994).

${ }^{13}$ M. Fukuma, M. Nagao, and M. Kosaki, Proceedings on 4th International Conference.on Properties and Applications of Dielectric Materials, Brisbane, 1994 (unpublished), pp. 24-27.

${ }^{14}$ K. Kaneko, Y. Suzuoki, and T. Mizutani, IEEE Trans. Dielectr. Electr. Insul. 6, 152 (1999).

${ }^{15}$ C. K. Williams, J. Electron. Mater. 21, 711 (1992). 